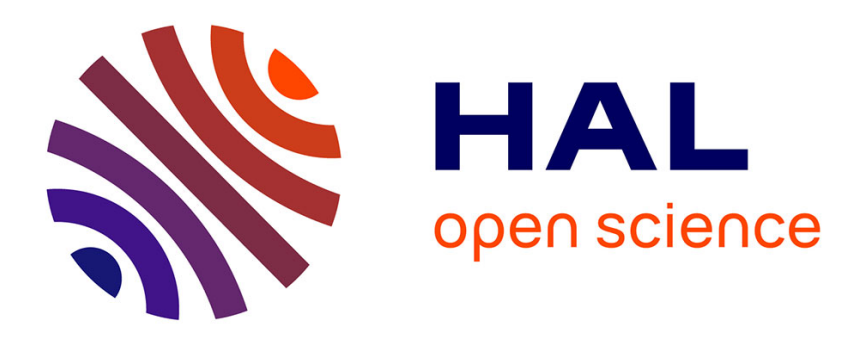

\title{
Défauts ponctuels dans les solides ferromagnétiques et ordre directionnel
}

\author{
Louis Néel
}

\section{To cite this version:}

Louis Néel. Défauts ponctuels dans les solides ferromagnétiques et ordre directionnel. Journal de Physique, 1963, 24 (7), pp.513-516. 10.1051/jphys:01963002407051300 . jpa-00205518

\section{HAL Id: jpa-00205518 https://hal.science/jpa-00205518}

Submitted on 1 Jan 1963

HAL is a multi-disciplinary open access archive for the deposit and dissemination of scientific research documents, whether they are published or not. The documents may come from teaching and research institutions in France or abroad, or from public or private research centers.
L'archive ouverte pluridisciplinaire HAL, est destinée au dépôt et à la diffusion de documents scientifiques de niveau recherche, publiés ou non, émanant des établissements d'enseignement et de recherche français ou étrangers, des laboratoires publics ou privés. 


\title{
DÉFAUTS PONCTUELS DANS LES SOLIDES FERROMAGNÉTIQUES ET ORDRE DIRECTIONNEL
}

\author{
Par M. Louis NÉEL, \\ Faculté des Sciences, Grenoble.
}

\begin{abstract}
Résumé. - Les méthodes utilisant les effets liés à l'ordre directionnel constituent des méthodes de choix pour étudier les défauts ponctuels produits par irradiation dans les solides ferromagnétiques : elles sont sensibles, spécifiques car chaque espèce de défaut se détecte dans une zone de température différente. De plus, elles permettent de déterminer la symétrie du défaut par rapport aux axes cristallins.

Abstract. - Methods connected with magnetic directional order are very suitable for studying point defects produced by irradiation of ferromagnetic solids : these methods are sensitive, and specific because each type of defect is detected in a specified temperature range. In addition, one can determine the symmetry of the defect with respect to the crystal axis.
\end{abstract}

L'étude des défauts ponctuels produits par irradiation dans les solides, dans les métaux par.exemple, est passionnante et difficile, en raison notamment du nombre et de la variété des défauts possibles. Il importe donc de trouver d'autres sources de renseignements que les méthodes classiques utilisant les anomalies de résistance électrique ou de chaleur spécifique et il est naturel de songer à des méthodes magnétiques.

On sait en effet que l'hystérésis ferromagnétique, tout au moins quand les cristallites ne sont pas trop petits, dépend presqu'exclusivement des défauts du réseau cristallin et est pratiquement absente quand il n'y a pas de défauts. En effet, tandis que l'aimantation à saturation ainsi que la température de Curie sont peu sensibles à la présence de défauts et que les lois d'approche à la saturation en fonction du champ magnétique sont un peu modifiées par la présence de défauts étendus comme les dislocations, l'amplitude et la forme du cycle d'hystérésis y sont extrêmement sensibles.

Le cycle d'hystérésis est défini par un grand nombre de paramètres dont les principaux sont le champ coercitif $H_{\mathrm{c}}$, le rapport $\boldsymbol{r}$ de l'aimantation rémanente $J_{\mathrm{r}}$ à l'aimantation à saturation $J_{\mathrm{s}}$, les coefficients $a$ et $b$ définissant la courbe de première aimantation dans les champs faibles $J=a H+b H^{2}$ (première loi de Rayleigh), la susceptibilité $J / H$ maximale $S_{\mathrm{m}}$, la susceptibilité anhystérétique initiale $i$, la susceptibilité différentielle $\mathrm{d} J / \mathrm{d} H$ maximale $S_{\text {dm }}$ le long du cycle limite.

En gros, la sensibilité aux défauts de ces différents paramètres croît dans l'ordre suivant $a, r, H_{\mathrm{c}}, b$, $S_{\mathrm{m}}, S_{\mathrm{dm}}, i$. C'est donc la susceptibilité anhystérétique initiale qu'il conviendrait de mesurer pour déceler dans les meilleures conditions l'apparition des défauts. Cette mesure est malheureusement difficile car il s'agit de déterminer l'aimantation obtenue dans un champ constant $H$, après appli- cation d'un champ alternatif lentement décroissant, jusqu'à zéro, à partir d'une valeur nettement supérieure à celle du champ coercitif.

En outre, puisqu'on désire étudier par des procédés magnétiques les défauts produits par les irradiations, il est préférable de prendre comme objet d'étude un échantillon initialement aussi parfait que possible, donc un échantillon magnétiquement doux. Dans de telles substances les variations d'aimantation proviennent principalement du déplacement des parois de Bloch séparant les domaines élémentaires et on sait alors qu'un défaut d'énergie totale donnée possède la plus grande. efficacité lorsque ses dimensions sont de l'ordre de grandeur des épaisseurs des parois, c'est-à-dire de quelques centaines à quelques milliers d'angströms. De ce point de vue, ce seront ainsi les défauts étendus qui joueront un rôle important : dislocations, joints de grains, cavités, inclusions, tensions internes microscopiques, etc... A priori, les défauts ponctuels, interstitiels, lacunes, etc... n'ont qu'une influence relativement faible sur les paramètres qui définissent l'hystérésis.

Une voie beaucoup plus féconde d'étude des défauts ponctuels paraît être celle de l'ordre directionnel, dont nous avons introduit le concept il y a dix ans.

Soit une solution solide composée d'atomes A et B. Il existe trois catégories $\mathrm{AA}, \mathrm{AB}$ et $\mathrm{BB}$ de paires d'atomes voisins dont le nombre dépend de la concentration $C_{\mathrm{A}}$ et $C_{\mathrm{B}}$ des atomes $\mathrm{A}$ et $\mathrm{B}$ et de l'existence éventuelle d'un ordre à courte distance. Une paire est en outre caractérisée par la direction $D$ de la droite joignant les centres des deux atomes constituants. Généralement, la probabilité $\varpi_{\mathrm{AA}} \mathrm{d} \omega$ de trouver la direction d'une paire de catégorie AA, par exemple, comprise dans un angle solide $d \omega$, centré autour d'une direction $\Delta$, ne dépend pas de l'orientation de $\Delta$ : il n'y a pas d'ordre directionnel. 
Cependant, dans une substance ferromagnétique, il faut attacher à chaque paire une certaine énergie qui dépend de l'angle $\theta$ de $D$ avec l'aimantation spontanée $J_{\mathrm{s}}$ : par exemple dans le cas le plus simple $\omega_{A A} \cos \theta$. Il en résulte qu'à l'équilibre $\varpi_{A A}$ dépend de $\theta:$ l'alliage possède un ordre directionnel à courte distance. Généralement, $N \omega_{\mathrm{AA}}$, où $N$ est le nombre d'Avogadro, est de l'ordre de $10^{6}$ à $10^{8}$ ergs tandis que $R T$ à $100^{\circ} \mathrm{K}$ est de l'ordre de $10^{10}$ ergs. Cet ordre directionnel est donc très peu accusé et les modifications relatives de $\varpi_{A A}$ avec $l^{2}$ orientation qui lui sont liées sont inférieures à un pour cent.

Pour que des modifications de l'ordre directionnel puissent se produire, il faut que la diffusion des atomes soit possible. Soit alors $T_{B}$ la température, dite température de blocage, à laquelle la diffusion s'effectue à l'échelle de quelques distances interatomiques pendant un intervalle de temps correspondant à la durée d'une expérience de laboratoire et supposons que $T_{\mathrm{B}}$, qui est de l'ordre de $700 \mathrm{oK}$ pour le fer, soit inférieur au point de Curie $\theta$ de l'alliage. Le domaine des températures peut alors être partagé en plusieurs intervalles.

Dans l'intervalle compris entre $T_{\mathrm{B}}$ et $\theta$, les variations d'ordre directionnel accompagnent les variations d'orientation de l'aimantation d'une manière pratiquement instantanée. Au voisinage de $T_{\mathbf{B}}$, les variations d'ordre directionnel suivent avec un certain retard les variations d'aimantation: ce sont les phénomènes du trâ̂nage magnétique de diffusion. Enfin, au dessous de $T_{\mathrm{B}}$, l'ordre directionnel reste invariable et n'est plus modifié par les variations d'orientation de l'aimantation spontanée : il conserve la valeur qu'il avait au moment où pour la dernière fois la température de l'échantillon s'est abaissée au-dessous de $T_{\mathrm{B}}$. En particulier lorsqu'on refroidit la substance dans un champ $H$, en imposant ainsi à l'aimantation $J_{\mathrm{s}}$ la direction de $H$, on provoque l'apparition d'un ordre directionnel et d'une anisotropie qui persiste sans altérations tant que la température ne remonte pas au dessus de $T_{\mathbf{B}}$ : ce sont les phénomènes d'orientation magnétique. Ils sont très faciles à mettre en évidence grâce à l'extrême sensibilité des mesures d'anisotrōpie magnétique à la bälance de torsion : presque $1 \mathrm{erg} / \mathrm{cm}^{3}$. Ils ont reçu de nombreuses applications dans le traitement thermique de certain's permalloys ou pour donner à des couches minces magnétiques des cycles d'hystérésis rectangulaires en vue de leur utilisation comme mémoires magnétiques.

En ce qui concerne le traînage magnétique de diffusion, au voisinage de la température $T_{\mathrm{B}}$, plusieurs méthodes d'observation sont possibles. On peut par exemple déterminer l'angle de perte, c'est-à-dire en champ magnétique alternatif le retard de phase de l'aimantation par rapport au champ magnétique. Cependant, l'étude des phéno- mènes de désaccommodation est plus riche en enseignements. Voici en quoi elle consiste. Au cours d'une désaimantation en champ alternatif décroissant, l'aimantation spontanée en chaque point s'oriente successivement, pendant un petit intervalle de temps, suivant un grand nombre de directions différentes, si bien qu'en moyenne l'aimantation spontanée est nulle et que tout ordre directionnel disparaît. Il en résulte que si nous traçons une courbe d'aimantation, aussitôt après la désaimantation, nous avons affaire à une substance isotrope, l'aimantation est facile et la perméabilité est grande.

"Si au contraire, nous laissons la substance séjourner dans un champ magnétique nul, l'aimantation spontanée en chaque point reste dans la même position et un ordre directionnel s'établit, diminuant l'énergie libre du système et contribuant à stabiliser dans leur état initial le système des domaines élémentaires et de leurs parois de séparation. L'échantillon s'aimante d'autant plus difficilement qu'on -a laissé s'écouler plus de temps depuis la désaimantation. Comme nous le verrons pous loin, les variations correspondantes de perméabilité peuvent être extrêmement importantes.

Comme l'a montré P. Brissonneau, l'étude détaillée de ces phénomènes de désaccommodation apporte des renseignements précieux sur la nature des atomes interagissants magnétiquement. Supposons qu'il s'agisse d'une solution solide, cubique, centrée comme le fer et dans laquelle les directions de facile aimantation, c'est-à-dire les directions d'aimantation spontanée des domaines élémentaires, soient les 3 axes quaternaires. Si les interactions magnétiques sont dues seulement aux huit atomes premiers voisins situés sur l'un des quatre axes ternaires, les directions des paires sont celles des axes ternaires. D'autre part, si on provoque une variation d'aimantation assez grande pour qu'on puisse négliger l'épaisseur des parois vis-àvis de leur déplacement total, le bilan final de cette opération consiste simplement à faire passer l'aimantation spontanée, en quelques régions, d'un certain axe quaternaire à un autre axe quaternaire. Mais les paires, étant orientées suivant un axe ternaire, sont toujours orientées symétriquement par rapport aux trois axes quaternaires. Il en résulte que pour les grandes variations d'aimantation, il n'y aura pas de désaccommodation de la perméabilité : ces variations resteront faciles.

Au contraire, pour de petites variations d'aimantation pour lesquelles l'amplitude des déplacements de paroi est égale ou inférieure à leur épaisseur, il peut y avoir désaccommodation puisqu'à l'intérieur de la paroi l'aimantation spontanée est par exemple parallèle à un axe binaire et qu'un axe binaire n'est pas orienté symétriquement par rapport aux axes ternaires. Une paroi peut ainsi, lorsque le temps s'écoule, se creuser un trou de 
potentiel dont l'épaisseur est de l'ordre de grandeur de sa propre épaisseur. Ces petites variations d'aimantation deviennent donc difficiles, mais dès que la paroi a franchi un intervalle égal à son épaisseur, comme il n'y a plus d'opposition au mouvement, l'aimantation varie d'une manière discontinue.

On montre de la même façon que s'il existe des interactions entre un atome et ses six seconds voisins, correspondant à des directions de paires parallèles aux axes quaternaires et qui ne sont plus symétriques par rapport aux trois axes quaternaires, une désaccommodation apparaît pour de grandes valeurs de la variation d'aimantation.

Finalement l'étude attentive des courbes de traînage magnétique permet de séparer quantitativement les effets des paires orientées suivant les axes quaternaires ou ternaires. Les figures 1 et 2,

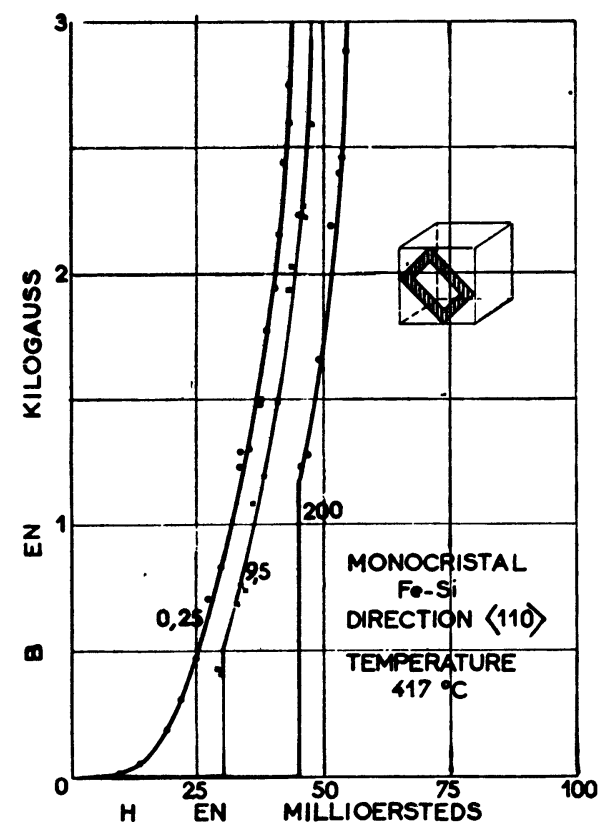

Fig. 1. - Aux grandes inductions, le traînage constant est attribué aux interactions entre atomes seconds voisins.

empruntées à Brissonneau montrent par exemple les courbes d'aimantation d'un monocristal de fersilicium, à $417^{\circ} \mathrm{C}$. La courbe marquée 0,25 est tracée $0,25 \mathrm{mn}$ après la désaimantation, l'aimantation est facile. Si on attend 1,5, 4,5, 9,5, 29,5, $200 \mathrm{mn}$ après la désaimantation, l'aimantation devient de plus en plus difficile et il faut des champs de plus en plus grands pour déclencher une variation irréversible d'aimantation. La figure 2 (courbes 9,5 et 200) montre nettement que l'on ne rejoint pas la courbe 0,25 , ce qui traduit le fait qu'il y a encore une certaine opposition aux grandes variations d'aimantation et qu'il y a ainsi des

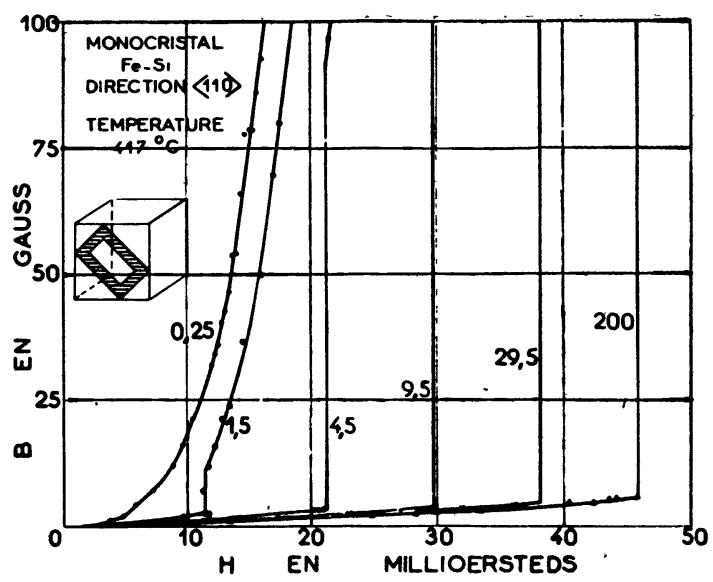

Fig. 2. - Aux faibles inductions, le décrochement a lieu pour un déplacement voisin du 1/4 de l'épaisseur de la paroi.

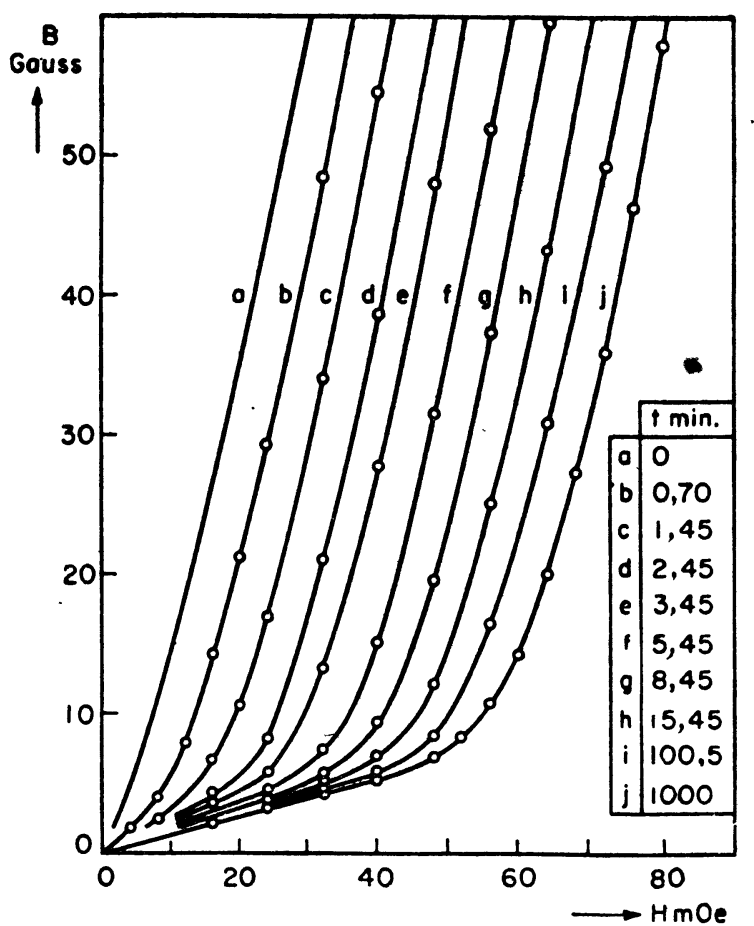

Fig. 3. - Courbes d'aimantation pour du fer contenant du carbone en position interstitielle $\left(4,6 \times 10^{-5}\right.$ en poids, à $10 \%$ près) à différentes étapes de la relaxation. On indique le temps de relaxation après désaimantation. La courbe a été obtenue par extrapolation. Température $245,9^{\circ} \mathrm{K}$.

interactions magnétiques correspondant aux paires de seconds voisins, paires parallèles aux axes quaternaires.

Tous ces phénomènes d'ord̛re directionnel se retrouvent dans un corps ferromagnétique, comme. le fer, contenant des atomes étrangers $\mathrm{X}$, comme 
du carbone, du bore, de l'azote, en position interstitielle, juste entre deux atomes de fer : proches voisins. Le rôle des paires est joué maintenant par des triades linéaires $\mathrm{Fe}-\mathrm{X}-\mathrm{Fe}$, parallèles à un des trois axes quaternaires.

La figure 3 , due à Brissonneau, montre par exemple les courbes de désaccommodation à $-27^{\circ} \mathrm{C}$ relatives à un échantillon ne contenant qu'un atome de carbone pour 20000 atomes de fer. La courbe d'aimantation a est tracée aussitôt après la désaimantation, la courbe $i, 100$ minutes après : l'effet est considérable. L'effet d'un atome de carbone pour $10^{6}$ atomes de fer serait encore décelable.

Ces atomes $B$ en substitution, ainsi que ces atomes étrangers interstitiels $X$ peuvent être considérés comme des défauts ponctuels : d'où l'idée d'étudier les défauts ponctuels produits par irradiation au moyen des effets liés à l'ordre directionnel.
Ces méthodes possèdent plusieurs avantages : elles sont sensibles. Elles sont spécifiques car à chaque défaut correspondent des températures $T_{\mathbf{B}}$ de blocage différentes. Elles permettent de déterminer la symétrie du défaut, caractéristique particulièrement précieuse pour l'identification des défauts.

Donnons quelques exemples relatifs aux défauts ponctuels dans le fer. Une lacune par exemple, type des défauts symétriques, ne doit pas provoquer d'ordre directionnel, tandis qu'un interstitiel dans le fer, symétrique par rapport à un axe quaternaire, est actif, mais les effets ne doivent pas être les mêmes que pour une dilacune ou une lacune piégée par un atome d'impureté en substitution qui sont des défauts symétriques par rapport à un axe ternaire.

Brissonneau et Moser ont développé ces noụvelles méthodes et en ont montré la fécondité. 